\title{
Detection of adenocarcinoma in Barrett's oesophagus by means of laser induced fluorescence
}

\author{
C Staël von Holstein, A M K Nilsson, S Andersson-Engels, R Willén, B Walther, \\ K Svanberg
}

\begin{abstract}
Patients-Seven patients with Barrett's metaplastic epithelium and oesophageal adenocarcinoma were investigated by means of laser induced fluorescence after low dose intravenous injection $(0.35 \mathrm{mg} / \mathrm{kg}$ bw) of Photofrin (QLT, Vancouver, Canada). Laser induced fluorescence measurements were performed immediately after resection of the oesophagus. Methods-Laser induced fluorescence spectra were recorded from 15-30 locations in each surgical specimen from normal mucosa, Barrett's epithelium, and tumour tissue. Histological examination was performed on each location to correlate the fluorescence spectral characteristics with histological status of the epithelium (normal, metaplastic or malignant). Measurements were also performed during endoscopy in five patients to test the applicability of the method in a clinical setting. Fluorescence spectra were recorded and evaluated at characteristic wavelengths, and biopsy specimens were collected. Fluorescence ratios were calculated as the quotient of Photofrin fluorescence divided by autofluorescence. Results-The mean (SD) fluorescence ratio values were $0.10(0.058)$ for normal oesophageal mucosa, $0.16(0.073)$ for normal gastric mucosa, $0.205(0.17)$ for Barrett's epithelium with moderate dysplasia, $0.79(0.54)$ for severe dysplasia, and $0.78(0.56)$ for adenocarcinoma. The highest fluorescence ratios were obtained for adenocarcinoma tissue, which could generally be distinguished from all nonmalignant tissue. Metaplastic Barrett's epithelium also yielded higher fluorescence ratios than did normal mucosa.

Conclusions-The results suggest that the technique can be used during endoscopy for real time tissue characterisation in the oesophagus, as an aid in detecting malignant transformation not macroscopically apparent at endoscopy. (Gut 1996; 39: 711-716)
\end{abstract}

Keywords: fluorescence diagnostics, Barrett's oesophagus, adenocarcinoma, low dose sensitisation, Photofrin, laser induced fluorescence.

Metaplastic columnar epithelium in the lower oesophagus - that is Barrett's oesophagus - is identified in about $10 \%$ of patients who under- go endoscopy for evaluation of symptoms of gastro-oesophageal reflux disease. ${ }^{1}$ The risk of developing oesophageal adenocarcinoma is 30-125 times greater among patients with Barrett's oesophagus than in the general population, ${ }^{23}$ and its incidence is increasing rapidly both in Europe and in the United States. ${ }^{4}$ Although few Barrett's cancers are detected at a stage when they are curable, and the overall five year survival rate is only about $7 \%,{ }^{6}$ if they are found at an early stage a cure is possible in almost all cases. ${ }^{8}$ However, early detection is difficult and early malignant changes are not accompanied by manifest symptomatology. ${ }^{9}$

Dysplasia constitutes a precursor lesion of adenocarcinoma, which develops in the metaplastic epithelium. ${ }^{3} 1011$ Thus, prospective endoscopic surveillance for the detection of high grade dysplasia or early adenocarcinoma has been recommended. However, there is significant inter and intra-observer variation in the distinction of different grades of dysplasia. ${ }^{12}$ Early malignant changes can often not be seen endoscopically, ${ }^{69}$ and the lesions are usually characterised by a patchy distribution within the metaplastic epithelium. ${ }^{13}$ Therefore, the possibility of sampling error during the biopsy procedure is substantial. A rigorous biopsy protocol, taking an average of 31 large samples at each endoscopy session, has been advocated as a means of increasing the probability of detecting early malignant changes. ${ }^{14}$ However, as such a biopsy protocol seems both difficult and impractical in a routine clinical setting, there is a pressing need to find other means of increasing the diagnostic yield in these patients. Laser induced tissue autofluorescence emission has been reported to discriminate between normal and $N$-nitrosomethyl-benzylamine induced oesophageal premalignant tissue in rats, ${ }^{15}$ and it was suggested that this technique might be used in humans for the detection of neoplasia both in the colon and in the oesophagus. ${ }^{16} 17$ Recently, a promising report on autofluorescence measurements in three cases of Barrett's adenocarcinoma was published. ${ }^{18}$ Earlier clinical in vivo results in tumour fluorescence detection were reported for various organs, such as the urinary bladder and the lungs. ${ }^{19}$ The applicability of low dose Photofrin fluorescence has been demonstrated. ${ }^{20}{ }^{21}$ Here we report the results of clinical fluorescence investigations using low dose Photofrin injection in patients with carcinoma and metaplastic epithelium of the oesophagus. To our knowledge, this is the first study of tumour 
marker enhanced laser induced fluorescence as an aid in detecting adenocarcinoma in Barrett's oesophagus.

\section{Methods}

PATIENTS AND HISTOLOGICAL EVALUATION The series comprised 12 patients, all injected intravenously with $0.35 \mathrm{mg} / \mathrm{kg}$ bw of Photofrin (QLT, Vancouver, Canada) 24 hours before the fluorescence measurements. The series was divided into two groups, group I (investigation on surgical specimens, $n=7$ ), and group II (measurements during endoscopy, $n=5$ ).

\section{Group I}

Group I consisted of six men and one woman, mean age 68 years (range, 57-77), operated on at University Hospital, Lund, for adenocarcinoma in Barrett's oesophagus. Total oesophagectomy with Ivor Lewis reconstruction was performed in all cases. The postoperative course was uneventful and all patients were discharged to their homes. Because of the low dose of the tumour marking agent, there were no cases of dermal photosensitisation, and no precautions concerning exposure to the sun or other light sources were necessary. ${ }^{20}{ }^{21}$

Surgical specimens were pinned to a cork plate, and laser induced fluorescence measurements were started immediately after the resection. In all, 149 fluorescence spectra were recorded from the resected specimens. All measurement sites were chosen from areas that looked typical to the naked eye for each type of mucosa. The optical fibre was kept in direct contact with the epithelium during the measurement and each measurement site was precisely marked with a needle. The measurement sites were numbered and a map containing all sites was drawn for the pathologist. After the laser induced fluorescence measurements, the specimen was fixed in $4 \%$ formaldehyde solution. Tissue from each measuring site was embedded in paraffin wax, cut in $4 \mu \mathrm{m}$

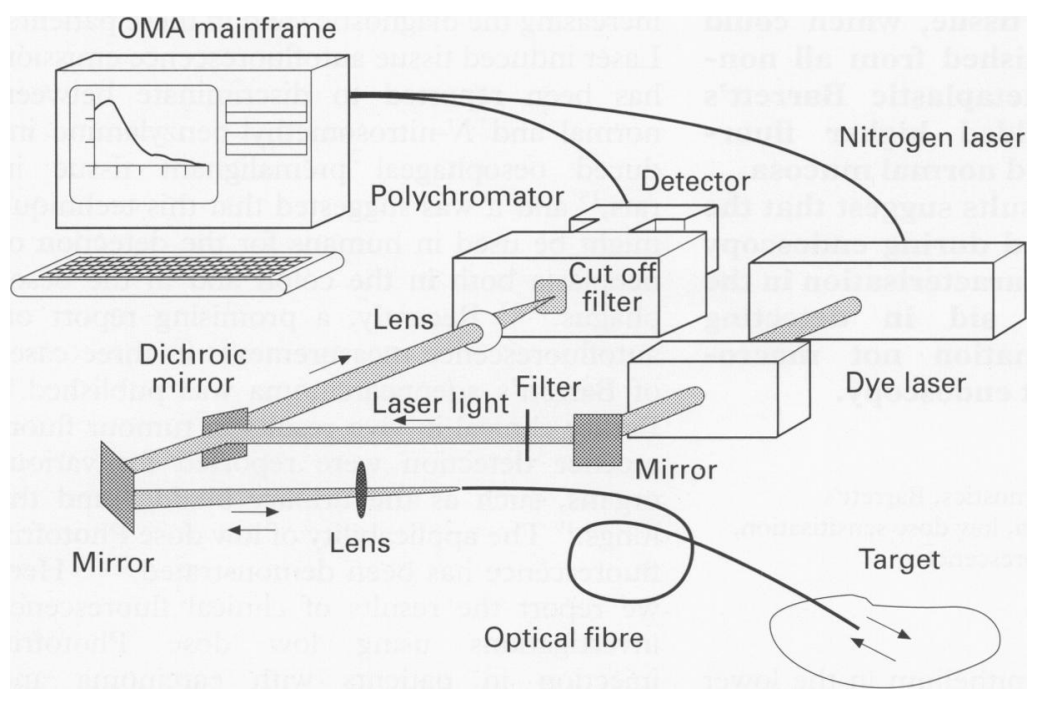

Figure 1: The set up of the optical multichannel analyser (OMA) system for fluorescence measurements. thick sections, and routinely stained with haematoxylin and eosin. For delineation of neutral and acid mucins, a combination of Alcian blue ( $\mathrm{pH} \mathrm{2.5)}$ and periodic acid Schiff mucin stains was applied. The track of the marking needle in the tissue was often present in the histological sections. Tissue from the measurement sites was evaluated and classified according to seven categories: normal oesophageal mucosa, normal gastric mucosa, Barrett's epithelium without dysplasia, mild dysplasia, moderate dysplasia, severe dysplasia or adenocarcinoma (carcinoma in situ included).

\section{Group II}

Group II consisted of five patients with Barrett's oesophagus in whom laser induced fluorescence measurements were performed during endoscopy. Three of the patients had shown severe carcinoma at endoscopy, histologically verified as adenocarcinoma. The other two patients had Barrett's oesophagus but no evidence of malignant transformation. The optical fibre transmitting laser light to and fluorescence from the tissue was introduced through the biopsy channel in the endoscope, being kept in close contact with the tissue during the laser induced fluorescence measurements. In all, 65 fluorescence spectra were recorded from the five patients. The tissue at the exact measurement site was assessed visually by the endoscopist, and final diagnosis of the patients was based on the collective evaluation of endoscopy and histology according to established practice. ${ }^{13}$

\section{FLUORESCENCE TUMOUR MARKER}

Photofrin is a fluorescent tumour marker used in conjunction with fluorescence diagnostics. The drug, composed of fluorescent porphyrins, is in general retained in tumour tissue to a higher degree than in normal tissue for more than 96 hours. The selective retention of porphyrins is used to distinguish malignant from normal tissue by analysing laser induced fluorescence signals. The porphyrins fluoresce with a characteristic bimodal distribution with peaks at about $630 \mathrm{~nm}$ and $690 \mathrm{~nm}$ wavelengths. Being porphyrin rich, malignant tissue thus differs in fluorescence spectra from normal tissue, which contains less porphyrins.

\section{EQUIPMENT FOR FLUORESCENCE}

MEASUREMENTS

Fluorescence measurements were performed with a clinically adapted optical multichannel analyser system arranged on a mobile trolley. The equipment has been described in detail elsewhere, ${ }^{19}$ and the set up is shown in Figure 1. Briefly, violet light (405 nm, 10 $\mu \mathrm{J} /$ pulse, $10 \mathrm{~Hz}$ ) was guided from a $\mathrm{N}_{2}$-laserpumped dye laser by mirrors, through lenses and finally through a $600 \mu \mathrm{m}$ core diameter quartz optical fibre to the target tissue, the fibre tip being kept in direct contact with the tissue examined. Some of the resulting fluorescence 


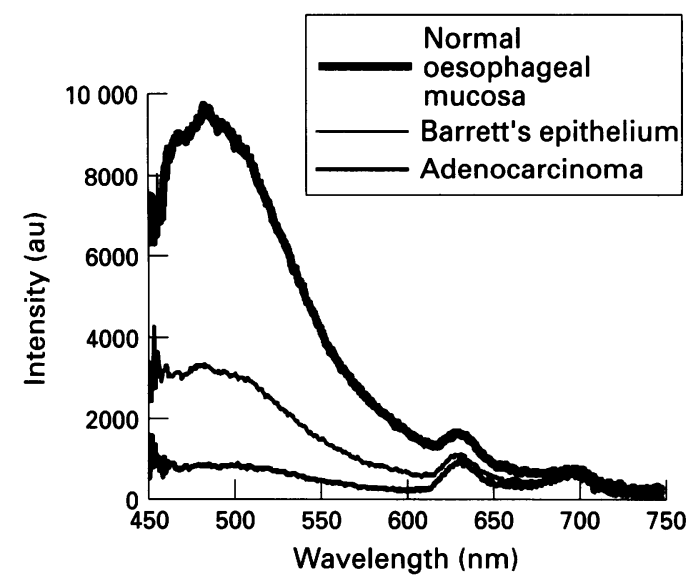

Figure 2: Typical fluorescence spectra recorded from normal oesophageal mucosa, Barrett's epithelium, and adenocarcinoma. The broad fluorescence peak in the spectra between 450 and $550 \mathrm{~nm}$ originates from endogenous tissue fluorophores (autofluorescence), while the two smaller peaks at about 630 and $700 \mathrm{~nm}$ are due to the low dose injection of Photofrin as a fluorescent tumour marker. The excitation wavelength was $405 \mathrm{~nm}$.

from the tissue and the retained porphyrin was collected by the same optical fibre and transmitted to a detector unit, a CCD (Charged Coupled Device) camera connected to a polychromator. In this way fluorescence spectra between 450 and $750 \mathrm{~nm}$ could be recorded with a resolution of $10 \mathrm{~nm}$. The equipment was controlled by computer. The optical fibre enabled the fluorescence measurements to be made during endoscopic examinations.

\section{ANALYSIS OF FLUORESCENCE SPECTRA}

Recorded fluorescence spectra contain two types of information in two different wavelength regions - a broad tissue autofluorescence peak at about $500 \mathrm{~nm}$ originating from endogenous tissue fluorophores and the bimodal porphyrin fluorescence with peaks above $600 \mathrm{~nm} .{ }^{19}$ Generally, the porphyrin fluorescence is stronger and the tissue autofluor-

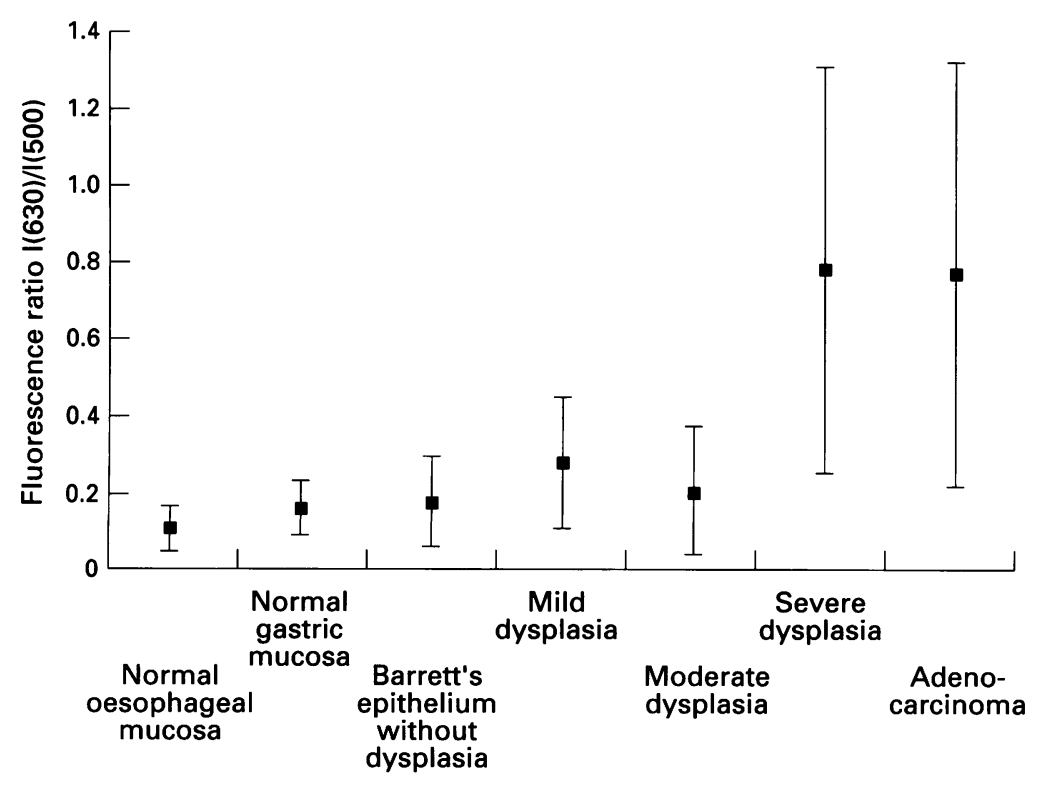

Figure 3: The mean (SD) values of the fluorescence ratios $(I(630 \mathrm{~nm}) / I(500 \mathrm{~nm}))$ obtained from the surgical specimens of patients in group $I$. escence weaker in malignant tissue than in normal mucosa. Hence, a tumour demarcation function was established in form of a fluorescence ratio: $\mathrm{I}(630 \mathrm{~nm}) / \mathrm{I}(500 \mathrm{~nm})$ - that is, the quotient of porphyrin fluorescence at 630 $\mathrm{nm}$ divided by autofluorescence at $500 \mathrm{~nm}$. Both fluorescence intensities utilised in this function thus contributed to tumour identification. By analysing the fluorescence spectra as dimensionless fluorescence ratios, the results were not sensitive to probe positioning, light source fluctuations or other measurement inconsistencies.

\section{Results}

Figure 2 shows three examples of recorded spectra, each typical of a different tissue type. The autofluorescence intensity at $500 \mathrm{~nm}$ in normal mucosa was on average 6.5 times higher than that for adenocarcinoma/severe dysplasia. The spectrum of Barrett's epithelium usually manifested an intermediate level of autofluorescence. Furthermore, the fluorescence intensity from Photofrin at 630 $\mathrm{nm}$ was on average only 1.2 times higher in tumour tissue than in normal tissue, though there was variation in this respect between samples, some of which did not manifest any difference in porphyrin fluorescence intensity between tumour tissue and surrounding normal mucosa.

Fluorescence ratio values from surgical specimens (group I) were divided into seven groups based on the histological evaluation of measurement sites: normal oesophageal mucosa (number of spectra, $n=35$ ), normal gastric mucosa $(n=25)$, Barrett's epithelium without dysplasia $(n=10)$, mild dysplasia $(n=18)$, moderate dysplasia $(n=7)$, severe dysplasia $(n=7)$, and adenocarcinoma including carcinoma in situ $(n=43)$. Four values were excluded, as the corresponding biopsy specimens contained both normal and malignant tissue. The mean (SD) fluorescence ratio values were $0.10(0.058)$ for normal oesophageal mucosa, $0 \cdot 16(0.073)$ for normal gastric mucosa, $0 \cdot 17(0 \cdot 12)$ for Barrett's epithelium without dysplasia, $0 \cdot 28(0 \cdot 17)$ for mild dysplasia, $0.205(0.17)$ for moderate dysplasia, $0.79(0.54)$ for severe dysplasia, and 0.78 $(0.56)$ for adenocarcinoma (Fig 3). Adenocarcinoma and severe dysplasia could be distinguished from normal gastric and oesophageal mucosa, whereas an overlap was found between fluorescence ratios from Barrett's epithelium including mild and moderate dysplasia on one hand and adenocarcinoma and severe dysplasia on the other, though ratios higher than 0.55 were obtained from adenocarcinoma or severe dysplasia in all cases. The results are also presented as the relative number of fluorescence spectra yielding a ratio in a certain well defined interval to evaluate better the distribution of the recorded fluorescence ratios for the different tissue types (Table). The first interval includes ratios lower than 0.16 (mean (SD) value for normal oesophageal mucosa), the second interval covers ratios between $0 \cdot 16$ and 0.375 (mean (SD) value for moderate 
Distribution of fluorescence ratios for the different tissue types. The scores given are the relative number, shown in percentage, of fluorescence spectra yielding ratios in the four intervals defined

\begin{tabular}{llllllll}
\hline $\begin{array}{l}\text { Relative number of } \\
\text { fluorescence ratios }\end{array}$ & $\begin{array}{l}\text { Normal } \\
\text { oesophageal } \\
\text { mucosa } \\
(n=35)\end{array}$ & $\begin{array}{l}\text { Normal } \\
\text { gastric } \\
\text { mucosa } \\
(n=25)\end{array}$ & $\begin{array}{l}\text { Barrett's } \\
\text { epithelium } \\
\text { without } \\
\text { dysplasia } \\
(n=10)\end{array}$ & $\begin{array}{l}\text { Mild } \\
\text { dysplasia } \\
(n=18)\end{array}$ & $\begin{array}{l}\text { Moderate } \\
\text { dysplasia } \\
(n=7)\end{array}$ & $\begin{array}{l}\text { Severe } \\
\text { dysplasia } \\
(n=7)\end{array}$ & $\begin{array}{l}\text { Adenocarcinoma } \\
(n=43)\end{array}$ \\
\hline $0<$ ratio<0.16 & $85 \cdot 7$ & 64 & 50 & $33 \cdot 3$ & $57 \cdot 1$ & 0 & 0 \\
$0 \cdot 16<$ ratio< $<0.375$ & $14 \cdot 3$ & 36 & 40 & $38 \cdot 9$ & $14 \cdot 3$ & $28 \cdot 6$ & $19 \cdot 5$ \\
$0 \cdot 375<$ ratio<0.55 & 0 & 0 & 10 & $27 \cdot 8$ & $28 \cdot 6$ & $14 \cdot 3$ & $26 \cdot 8$ \\
Ratio $>0 \cdot 55$ & 0 & 0 & 0 & 0 & 0 & $57 \cdot 1$ & $53 \cdot 7$ \\
\hline
\end{tabular}

dysplasia), the third interval contains ratios between 0.375 and the previously mentioned cut off value 0.55 , and the last interval includes ratios higher than 0.55 . Histological examination showed that 11 of 145 measurement sites had another epithelium than judged by the clinician. Two measurement sites with fluorescence ratios 0.085 and 0.14 were considered to be Barrett's epithelium by the clinician, but were judged by the pathologist to be normal mucosa. The fluorescence ratios for these sites were consistent with the pathological diagnosis. Another two measurement sites, both with fluorescence ratios $0 \cdot 25$, seemed to be malignant tissue, but were judged by the pathologist to be Barrett's epithelium with no or mild dysplasia - a diagnosis that again was in accordance with the fluorescence ratios obtained at these sites. The remaining seven measurement sites appeared to be normal Barrett's epithelium without malignancy to the naked eye, but they had fluorescence ratios with a mean value of 0.85 , ranging from 0.44 to $1 \cdot 43$, these values being highly suggestive of malignancy. At all of these sites, histological evaluation confirmed adenocarcinoma or severe dysplasia.

In the second part of the study we evaluated the possibility of performing measurements during endoscopy in three patients with Barrett's adenocarcinoma and two with Barrett's epithelium but no evidence of malignancy (group II). We used the ratio of 0.55 as a cut off level to distinguish malignant from other tissue types. Classification of measurement sites was based on visual and histological assessment according to normal clinical practice. There was very good overall agreement between the endoscopist's judgement of the measurement site and the respective fluorescence ratio. Of 17 fluorescence ratios from malignant tissue, 15 were above 0.55 ; and of 48 ratios from what was judged as normal mucosa or Barrett's epithelium 45 were below $0 \cdot 55$.

\section{Discussion}

Patients with Barrett's oesophagus represent a diagnostic challenge. Although endoscopic surveillance is widely recommended ${ }^{22}{ }^{23}$ it has many shortcomings. Unresectable malignancy may develop in patients undergoing regular surveillance, reliable markers of oesophageal malignant tumour are absent, the cost is substantial, and there is no proof that surveillance reduces mortality from oesophageal cancer. ${ }^{12}$ However, our results show the feasibility of using laser induced fluorescence to distinguish between normal and malignant tissue. The measurements can easily be made with an endoscope and seem to offer a solution to some diagnostic problems, although additional studies are needed before a clinical application can be recommended.

The shape of the recorded fluorescence spectrum was found to be characteristic of the tissue type from which it was obtained: normal mucosa, Barrett's epithelium or malignant tumour. The spectra from malignant tissue manifested a distinctly lower intensity of autofluorescence than did those from normal mucosa. The measurements from surgical specimens (group I) showed the mean (SD) fluorescence ratio, to distinguish normal oesophageal mucosa from malignant tumour tissue. As the ratios above 0.55 represented severe dysplasia or adenocarcinoma in all cases in our study, the probability is high that fluorescence ratios above 0.55 will represent malignancy. Furthermore, as no ratios representing severe dysplasia or adenocarcinoma were found below $0 \cdot 16$, the probability is low that fluorescence ratios below $0 \cdot 16$ will represent malignancy. It is more difficult to categorise ratios between 0.16 and 0.55 because of the overlap in ratio values between normal mucosa, Barrett's epithelium with and without dysplasia, and malignant tissue (Table). In general it could be said that higher ratios have higher probability to originate from malignant tissue. Superficial tumour necrosis or large amounts of blood or opalescent mucus over the tumour might compromise the results, due to the short penetration depth of the excitation light - in the order of half a millimetre. However, this is not a clinical problem as this occurs only in advanced and macroscopically manifest tumours. Our results show that thin, early malignant changes as carcinoma in situ and severe dysplasia, which are clinically difficult to identify with conventional methods, can be detected using fluorescence diagnostics.

We found the ratios obtained from normal gastric mucosa to be slightly higher than those from normal oesophageal mucosa. This may result from a difference in the structure of the epithelium - that is, columnar versus squamous epithelium. The higher ratios obtained for Barrett's epithelium without dysplasia might thus partly be explained by the columnar structure of the epithelium.

For the endoscopic surveillance a cut off level of 0.55 for fluorescence seemed appropriate as higher ratios were otained for all patients with malignancy, but not for patients 
with Barrett's oesophagus with no evidence of malignancy. Thus, diagnosis based on fluorescence measurements was in good accord with that based on biopsies. However, it is not possible to exactly pin point measurement sites of an area less than 1-2 $\mathrm{mm}^{2}$ with the biopsy forceps during endoscopy when the organ is moving and the optical fibre and biopsy forceps have to be introduced one after the other into the biopsy channel of the instrument. Evaluation of the reliability of this diagnostic technique must thus be based on the measurements on group I, where an exact histological correlation was obtained. The ratios obtained during endoscopy were mainly classified according to the visual assessment of the tissue. This classification is probably reliable regarding areas with manifest tumour, but in the three patients with malignant tumours we also obtained some high fluorescence ratios from areas that were visually normal. However, as early malignant changes are known to resemble normal Barrett epithelium, the possibility cannot be ruled out that these readings were from malignant tissue as well. In the two patients with Barrett's oesophagus but no evidence of malignancy, all ratios were below our cut off value and follow up so far has failed to reveal any malignant transformation.

In general, published studies of laser induced fluorescence in other organs have shown tumour marker signals to be significantly greater in malignant than in normal tissue, whereas in this study of Barrett's oesophagus Photofrin related signals in malignant tissue did not differ significantly from that in normal tissue. Thus, our ability to discriminate between malignant and normal tissue was mainly due to endogenous fluorophores (autofluorescence) and not the tumour specificity of the sensitising agent. Available evidence provides no clue to the reason why adenocarcinoma in the oesophagus should differ in this respect. The discriminative capacity of autofluorescence was better than anticipated, however, which implies that fluorescence diagnostics could be used for detection of malignant tumours in the oesophagus without the necessity of using any sensitiser, thus simplifying the procedure.

Although fluorescence diagnostics cannot replace biopsies and histopathology, it can guide the physician during endoscopy in selecting biopsy sites in suspicious areas indicated by high fluorescence ratios. If multiple biopsy specimens are taken from such an area, the probability of finding early malignant tumours is very high, as compared with the random biopsy approach. The study was performed with a prototype laboratory equipment with a rather slow computer, only permitting evaluation of one point at a time. Measurements done during endoscopy only allowed 30 sites to be evaluated during a 15 minute session. This is too slow in a routine clinical setting and makes it difficult to cover the whole area of Barrett's epithelium. There has now been further development of fibres and equipment, however, allowing the investigator to sweep the optical fibre for pathological measurements over the mucosa. Although still in a developmental phase this seems promising as it would permit screening of the entire Barrett's mucosa during a couple of minutes, guiding the endoscopist to take biopsy specimens from interesting areas. The price for such equipment has been calculated to about $£ 10000$. As fluoresence measurements pick up areas in a very early malignant phase, and as we know that the development from dysplasia to an established malignancy is a rather slow process, this might allow prolonged intervals between screening episodes, thus increasing the cost effectiveness of the procedure. A full image of the oesophagus in terms of fluorescence ratios, which has been done on a research basis in other organs, ${ }^{24}$ might further simplify the procedure.

In conclusion, Barrett's adenocarcinoma is a malignancy characterised by rapidly increasing incidence and dismal prognosis in advanced stages. $^{4-7}$ If these tumours are detected at an early stage, however, a cure can be obtained in almost all cases. ${ }^{8}$ The currently available technique - regular endoscopic surveillance with random biopsies in patients with Barrett's oesophagus - has many shortcomings. ${ }^{12}$ The search for new diagnostic tools to improve early discovery of this tumour is therefore of utmost importance. We have shown laser induced fluorescence to distinguish malignant tissue both from metaplastic and from normal epithelium in Barrett's oesophagus. Although additional studies have to be performed as some questions still remain to be answered, this diagnostic technique is promising and seems to have the potential to increase the diagnostic yield in Barrett's oesophagus.

Valuable discussions and support from Professor Sune Svanberg are appreciated. The study was funded by the Swedish Research Council for Engineering Sciences, the Swedish Cancer Foundation, and the Xillix Technologies Corp, which is gratefully acknowledged.

1 Spechler S, Goyal R. Barrett's oesophagus. $N$ Engl f Med 1986; 315: 362-71.

2 Cameron A, Ott B, Payne W. The incidence of adenocarcinoma in columnar-lined (Barrett's) oesophagus. $N$ Engl f Med 1985; 313: 857-9.

3 Hameeteman W, Tytgat G, Houthoff $\mathrm{H}$, van den Tweel J. Barrett's oesophagus. Development of dysplasia and Barrett's oesophagus. Development of dysplasia and

4 Hansson L, Sparén P, Nyrén O. Increasing incidence of both major histological types of oesophageal carcinomas both major histological types of oesophageal carcinom

5 Pera M, Cameron A, TrasteckV, Carpenter H, Zinsmeister A Increasing incidence of adenocarcinoma of the oesophagus and oesophagogastric junction. Gastroenterology 1993; 104: 510-3.

6 Skinner D, Walther B, Riddell R, Schmidt H, Iascone C DeMeester T. Barrett's oesophagus: comparison of benign and malignant cases. Ann Surg 1983; 198: 554-66.

7 Sjögren R, Johnsson L. Barrett's oesophagus: A review. Am fMed 1983; 74: 313-20.

8 Johansson J, Johnsson F, Walther B, Staël von Holstein C, Willén $R$, Zilling T. Adenocarcinoma in the distal oesophagus with and without Barrett's epithelium. Differences in symptoms and survival rate. Arch Surg 1996, 131: ences in sym.

9 Robertson C, Mayberry J, Nicholson D, James P, Atkinson M. Value of endoscopic surveillance in the detection of neoplastic change in Barrett's oesophagus. $B r \mathcal{f}$ Surg 1988; 75: 760-3.

10 Schmidt H, Riddell R, Walther B, Skinner D, Riemann J. Dysplasia in Barrett's oesophagus. $\mathcal{F}$ Cancer Res Clin Oncol 1985; 110: 145-52.

11 Miros M, Kerlin P, Walker N. Only patients with dysplasia progress to adenocarcinoma in Barrett's oesophagus. Gut 1991; 32: 1441-6.

12 Tytgat G. Does endoscopic surveillance in oesophagea columnar metaplasia (Barrett's oesophagus) have any real value? Endoscopy 1995; 27: 19-26. 
13 Reid B, Weinstein W, Lewin K, Haggitt R, van Deventer G, den Besten $\mathrm{L}$, et al. Endoscopic biopsy can detect highgrade dysplasia or early adenocarcinoma in Barrett's grade dysplasia or early adenocarcinoma in Barrett's oesophagus without grossly recognisab

14 Levin D, Haggitt R, Bluont P, Rabinovitch P, Rusch V, Reid B. An endoscopic biopsy protocol can differentiate highgrade dysplasia from early adenocarcinoma in Barrett's oesophagus. Gastroenterology 1993; 105: 40-50.

15 Glasgold R, Glasgold M, Savage H, Pinto J, Alfano R, Schantz S. Tissue autofluorescence as an intermediate endpoint in NMBA-induced oesophageal carcinogenesis. Cancer Lett 1994; 82: 33-41.

16 Kapadia C, Cutruzola F, O'Brien K, Stetz M, Enriques R, Deckelbaum L. Laser-induced fluorescence spectroscopy of human colonic mucosa: detection of adenomatous transformation. Gastroenterology 1990; 99: 150-7.

17 Vo-Dinh T, Panjehpour M, Overholt B, Farris C, Buckley III $P$, Sneed R. In vivo cancer diagnostis of the oesophagus using differential normalized fluorescence oesophagus using differential normaliz

18 Wang K, Gutta K, Laukka M, Densmore J. Laser induced fluorescence in the detection of oesophageal carcinoma. SPIE 1995; 2324: 14-8.
19 Andersson-Engels S, Elner $\AA$, Johansson J, Karlsson S-E, Salford LG, Strömblad L-G, et al. Clinical recordings of laser-induced fluorescence spectra for evaluation of tumour demarcation feasibility in selected clinical tumour demarcation feasibility in selected

20 Baert L, Berg R, van Damme B, Johansson J, Svanberg K, Svanberg S. Clinical fluorescence diagnosis of human bladder carcinoma following low-dose Photofrin injection. Urology 1993; 41: 322-30.

21 Lam S, Palcic B, McLean D, Hung J, Korblik M, Profio AE. Detection of early lung cancer using low dose Photofrin II. Chest 1990; 97: 333-7.

22 American Society of Gastrointestinal Endoscopist: Statements and guidelines developed by the standards of the Training and Practice committee of the ASGE: the role of endoscopy in the surveillance of premalignant conditions of the in the surveillance of premalignant conditions

23 Dent J, Bremner C, Collen M, Haggitt R, Spechler S. Barrett's oesophagus. Working party reports of the world congress of gastroenterology. 9th Congress of Gastrocongress of gastroenterology. 9th Congress

24 Andersson-Engels S, Johansson J, Svanberg S. Medical diagnostic system based on simultaneous multi-spectral fluorescence imaging. Applied Optics 1994; 33: 8022-9. 